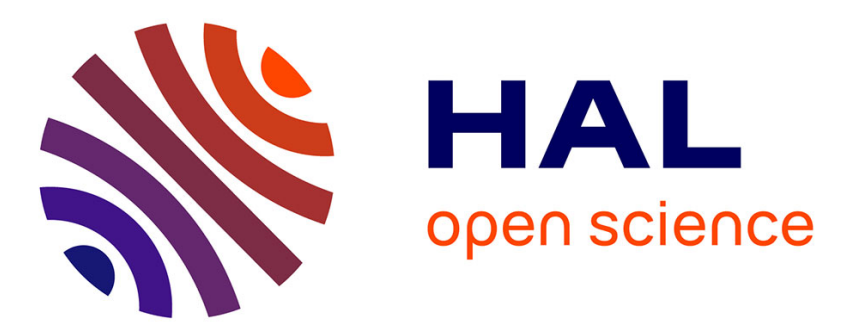

\title{
Discrete mode observability of structured switching descriptor linear systems: A graph-theoretic approach
}

Taha Boukhobza, Frédéric Hamelin

\section{To cite this version:}

Taha Boukhobza, Frédéric Hamelin. Discrete mode observability of structured switching descriptor linear systems: A graph-theoretic approach. Automatica, 2013, 49 (10), pp.3042-3048. 10.1016/j.automatica.2013.06.006 . hal-00853675

\section{HAL Id: hal-00853675 https://hal.science/hal-00853675}

Submitted on 23 Aug 2013

HAL is a multi-disciplinary open access archive for the deposit and dissemination of scientific research documents, whether they are published or not. The documents may come from teaching and research institutions in France or abroad, or from public or private research centers.
L'archive ouverte pluridisciplinaire HAL, est destinée au dépôt et à la diffusion de documents scientifiques de niveau recherche, publiés ou non, émanant des établissements d'enseignement et de recherche français ou étrangers, des laboratoires publics ou privés. 


\title{
Discrete mode observability of structured switching descriptor linear systems: a graph-theoretic approach
}

\author{
T. Boukhobza and F. Hamelin \\ Université de Lorraine, CRAN, UMR 7039, Campus Sciences, BP 70239, Vandoeuvre-les-Nancy Cedex, 54506, France \\ email: taha.boukhobza@univ-lorraine.fr
}

\begin{abstract}
The main result of the paper consists in graphical necessary and sufficient conditions which ensure the generic discrete mode observability of structured switching descriptor systems. The methods used in the previous studies on the observability of switching linear systems on standard form are not applicable to switching descriptor systems. So, we develop a new approach starting from bipartite representations of these systems and then building a new kind of digraph dedicated to the discrete mode observability study. The proposed method assumes only the knowledge of the system's structure and is applicable to a large class of descriptor systems including regular and non regular systems even if they are square or under-determined. The provided conditions can be implemented by classical graph-theory algorithms. Key words: Switching systems, descriptor systems, mode observability, graph theory.
\end{abstract}

\section{Introduction}

Hybrid systems, combining event-driven and time-driven dynamics, have received growing attention in the control community as they describe a wide range of systems (Johansson and Rantzer (Eds), 2007). On the other hand, descriptor systems, which handle systems with both differential and non-differential relations, result from a convenient and natural modelling process (Müller, 2000). Their applications can be found in robotics, electrical networks, biologic and economic systems (Müller, 2000). When the model representing the whole or more generally a part of a system is a singular model (for modelling convenience), the functioning system is then represented by a switching descriptor system and in order to check the functioning mode, we have to observe the discrete mode variable of the switching system. Switching descriptor systems are also particularly suited to handle systems (even in standard form) where the dynamics of the continuous part is not entirely known in each discrete mode. Some practical examples where the switching descriptor models are useful and pertinent are provided in (Boukas, 2008; Clotet et al., 2009; De Koning, 2003). The paper focuses on the discrete mode observability of switching descriptor systems. The discrete mode observability is relevant to detect some abrupt changes due to faults and which make the system switching to non nominal dynamics or for supervision when the switching between different modes implies control structure modifications. Few works deal with the observability of switching descriptor system, whereas the developed approaches used to study systems in standard form are not directly applicable. Moreover, for the most part, observability studies use algebraic or geometric approaches and so require the exact knowledge of the state space matrices characterizing the systems' model. In many modelling problems or during the conception stage, these matrices have a number of fixed zero entries determined by the physical laws while the remaining entries are not precisely known. In these cases, to study the structural properties, like observability, the idea is that we only keep the zero/non-zero entries in the state space matrices. Many interesting works on these models, called structured models, aim to analyse their properties (Dion $e t$ al., 2003; Murota, 1987; Reinschke, 1988).
The paper is organised as follows: after Section 2, which is devoted to the problem formulation, some definitions related to the graph-theoretic approach are given in Section 3 . The main result is provided in Section 4 before a brief conclusion.

\section{Problem statement}

Consider the following switching descriptor system (SDS)

$$
\Sigma:\left\{\begin{aligned}
E_{r(t)} \dot{x}(t) & =A_{r(t)} x(t) \\
y(t) & =C_{r(t)} x(t)
\end{aligned}\right.
$$

where $x \in \mathbb{R}^{n}$ and $y \in \mathbb{R}^{p}$ are respectively the state vector and the output (measurement) vector and where $E_{r(t)} \in$ $\mathbb{R}^{m \times n}, A_{r(t)} \in \mathbb{R}^{m \times n}$ and $C_{r(t)} \in \mathbb{R}^{p \times n}$. In order to guarantee that there exists at least one trajectory $x(t)$ satisfying the relations defining system $\Sigma, E_{r(t)} x\left(0_{-}\right)$is assumed to be admissible i.e. it does not result in contrary equations in $\Sigma$ and is such that system $\Sigma$ is solvable. The exogenous and unobserved discrete mode variable (or switching signal) $r:[0, \infty) \rightarrow Q=\{1, \ldots, N\}$, is assumed, as in (Babaali and Pappas, 2005), to be right-continuous and only a finite number of jumps can occur in any finite interval.

The discrete mode observability is the capacity to deduce the discrete mode knowing the measurements. It is based on the mode distinguishability:

Definition 1 (Mode distinguishability) Two distinct modes $q \in Q$ and $q^{\prime} \in Q$ are distinguishable if, for almost all initial conditions $x_{0}$, either there exist an integer $s \geq 0$ and an expression $f_{q}\left(y, \dot{y}, \ldots, y^{(s)}\right)=0$ which is satisfied for mode $q$ but is not satisfied for mode $q^{\prime}$, or there exist an integer $s^{\prime} \geq 0$ and an expression $f_{q^{\prime}}\left(y, \dot{y}, \ldots, y^{\left(s^{\prime}\right)}\right)=0$ which is satisfied for mode $q^{\prime}$ but is not satisfied for mode $q$. Here, "for almost all initial conditions $x_{0}$ " is to be understood as "for all $x_{0} \in \mathbb{R}^{n}$ except for the zero set of some polynomials with real coefficients in the $n$ initial state components" ( $x_{0}=0$ for example).

Definition 2 (Discrete mode observability) $\Sigma$ is discrete mode observable if its modes are distinguishable 2-by-2.

Discrete mode observability analysis can then be reduced to the study of the distinguishability of each pair of modes. 
Thus, in this paper, we consider that we have only 2 modes. Moreover, since we study a structural property, it is pertinent to deal with structured systems, for which we assume that only the sparsity pattern of matrices $E_{q}, A_{q}$ and $C_{q}$ is known for $q \in\{1,2\}$. So, to each entry of these matrices, we only know whether its value is fixed to zero, or that it has an non-fixed real value represented by a parameter $\lambda_{i}$. The vector of these parameters is $\Lambda=\left(\lambda_{1}, \lambda_{2}, \ldots, \lambda_{h}\right)^{T}$ and it is assumed that $\Lambda$ can take any value in $\mathbb{R}^{h}$. We denote by $A_{q}^{\lambda}, C_{q}^{\lambda}$ and $E_{q}^{\lambda}$ respectively the matrices obtained by replacing the non zeros in $A_{q}, C_{q}$ and $E_{q}$, for $q \in\{1,2\}$ by the corresponding parameters $\lambda_{i}$ and we denote

$$
\Sigma_{\Lambda}:\left\{\begin{aligned}
E_{r(t)}^{\lambda} \dot{x}(t) & =A_{r(t)}^{\lambda} x(t) \\
y(t) & =C_{r(t)}^{\lambda} x(t)
\end{aligned}\right.
$$

If all parameters $\lambda_{i}$ are numerically fixed, we obtain a socalled admissible realization of $\Sigma_{\Lambda}$. We say that a property is true generically for $\Sigma_{\Lambda}$ if it is true for almost its realizations or equivalently for almost all parameters $\lambda_{i}$.

For the discrete mode observability analysis, it is pertinent and necessary to highlight the similarities and the differences between the models associated to these modes. Indeed, for $q \neq q^{\prime}$, it is not realistic to assume that all the parameters of $A_{q}^{\lambda}, C_{q}^{\lambda}$ or $E_{q}^{\lambda}$ are free from the ones of $A_{q^{\prime}}^{\lambda}, C_{q^{\prime}}^{\lambda}$ or $E_{q^{\prime}}^{\lambda}$. Thus, we decompose each structured matrix into 2 parts: the first one is common to the 2 modes and the second one is specific to each mode i.e. for $q \in\{1,2\}, A_{q}^{\lambda}=$ $A_{0}+A_{q}^{s}, C_{q}^{\lambda}=C_{0}+C_{q}^{s}$ and $E_{q}^{\lambda}=E_{0}+E_{q}^{s}$. We assume that the entries of these matrices are free and that a coefficient of $A_{q}^{\lambda}$ (resp. $C_{q}^{\lambda}$ and $E_{q}^{\lambda}$ ) is exclusively in $A_{0}$ or in $A_{q}^{s}$ (resp. in $C_{0}$ or in $C_{q}^{s}$, and in $E_{0}$ or in $E_{q}^{s}$ ). These notations can be extended to the multi-mode case (Boukhobza and Hamelin, 2011).

\section{Graphical representation and definitions}

For each mode $q=1,2$, we associate to structured system $\Sigma_{\Lambda}$ a bipartite graph noted $B\left(\Sigma_{\Lambda}, q\right)=\left(\mathbf{V}^{+}, \mathbf{V}^{-}, W_{q}\right)$, where $\mathbf{V}^{+}$and $\mathbf{V}^{-}$are 2 disjoint vertex subsets and $W_{q}$ is the edge set related to mode $q$. The vertices are associated to the whole internal state, dynamical variables and outputs of $\Sigma_{\Lambda}$ and the edges represent links between these variables for each mode. More precisely, $\mathbf{V}^{+}=\mathbf{X}$ and $\mathbf{V}^{-}=\mathbf{Y} \cup \mathbf{Z}$, with $\mathbf{X}=\left\{\mathbf{x}_{\mathbf{1}}, \mathbf{x}_{\mathbf{2}}, \ldots, \mathbf{x}_{\mathbf{n}}\right\}, \mathbf{Z}=\left\{\mathbf{z}_{\mathbf{1}}, \mathbf{z}_{\mathbf{2}}, \ldots, \mathbf{z}_{\mathbf{m}}\right\}$ representing relation $z=E_{r(t)}^{\lambda} x$ and $\mathbf{Y}=\left\{\mathbf{y}_{\mathbf{1}}, \mathbf{y}_{\mathbf{2}}, \ldots, \mathbf{y}_{\mathbf{p}}\right\}$. Edge set is related to each mode $q$ and is defined by $W_{q}=A_{q}$-edges $\cup C_{q^{-}}$edges $\cup E_{q}$-edges, where $A_{q}$-edges $=$ $\left\{\left(\mathbf{x}_{\mathbf{j}}, \mathbf{x}_{\mathbf{i}}\right) \mid A_{q}^{\lambda}(i, j) \neq 0\right\}, C_{q}$-edges $=\left\{\left(\mathbf{x}_{\mathbf{j}}, \mathbf{y}_{\mathbf{i}}\right) \mid C_{q}^{\lambda}(i, j) \neq 0\right\}$ and $E_{q}$-edges $=\left\{\left(\mathbf{x}_{\mathbf{j}}, \mathbf{z}_{\mathbf{i}}\right) \mid E_{q}^{\lambda}(i, j) \neq 0\right\}$. Each edge is associated to a free non-zero parameter of the system's model called the weight of the edge. Number $q$ is written under each edge associated to an element of specific matrices $A_{q}^{s}, C_{q}^{s}$ and $E_{q}^{s}$ and represents its index. The edges which are common to the two modes i.e. associated to matrices $A_{0}, C_{0}$ and $E_{0}$ have index 0 . The edges which are specific to mode $q$ have index $q$.

Example 1 To the system defined by the following matrices, we associate bipartite graphs in Figure 1.
$A_{0}=\left(\begin{array}{cccccc}0 & \lambda_{1} & 0 & 0 & 0 & 0 \\ 0 & 0 & 0 & 0 & 0 & \lambda_{2} \\ 0 & 0 & 0 & \lambda_{3} & 0 & 0 \\ 0 & 0 & 0 & 0 & \lambda_{4} & 0\end{array}\right), C_{0}=\left(\begin{array}{cccccc}\lambda_{5} & 0 & 0 & 0 & 0 & 0 \\ 0 & \lambda_{6} & 0 & 0 & 0 & 0 \\ 0 & 0 & \lambda_{7} & 0 & 0 & 0 \\ 0 & 0 & 0 & \lambda_{8} & 0 & 0\end{array}\right), E_{0}=\left(\begin{array}{cccccc}\lambda_{9} & 0 & 0 & 0 & 0 & 0 \\ 0 & 0 & 0 & 0 & 0 & \lambda_{10} \\ 0 & \lambda_{11} & \lambda_{12} & 0 & 0 & 0 \\ 0 & 0 & 0 & \lambda_{13} & 0 & 0\end{array}\right)$, The specific matrices for mode 1 are such that the entries of $A_{1}^{s}$ are zero except $A_{1}^{s}(3,1)=\lambda_{14}, C_{1}^{s}=0$ and the entries of $E_{1}^{s}$ are zero except $E_{1}^{s}(2,3)=\lambda_{15}$. The specific matrices for mode 2 are such that $A_{2}^{s}=0, C_{2}^{s}=0$ and the entries of $E_{2}^{s}$ are zero except $E_{2}^{s}(3,1)=\lambda_{16}$.
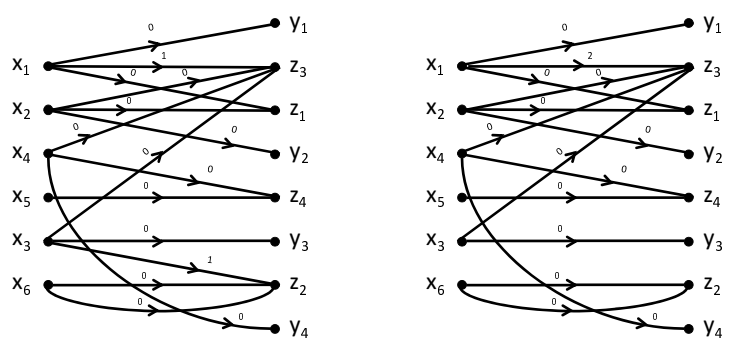

Figure 1. Bipartite graphs associated to system of Example 1. Mode 1 on the left, mode 2 on the right.

- Two edges are disjoint if they have no common vertex. A matching is a set $M$ of disjoint edges.

- A path $P$ is denoted $P=\mathbf{v}_{\mathbf{s}_{\mathbf{0}}} \rightarrow \mathbf{v}_{\mathbf{s}_{1}} \rightarrow \ldots \rightarrow \mathbf{v}_{\mathbf{s}_{\mathbf{i}}}$, where, for a given $q \in\{1,2\},\left(\mathbf{v}_{\mathbf{s}_{\mathbf{j}}}, \mathbf{v}_{\mathbf{s}_{\mathbf{j}+\mathbf{1}}}\right) \in W_{q}$ for $j=0,1, \ldots, i-1$. We say in this case that $P$ covers $\mathbf{v}_{\mathbf{s}_{\mathbf{0}}}, \mathbf{v}_{\mathbf{s}_{1}}, \ldots, \mathbf{v}_{\mathbf{s}_{\mathbf{i}}}$. A path is simple when every vertex occurs only once. The weight of $P$ is the product of the weights of all its edges. A cycle is a path of the form $\mathbf{v}_{\mathbf{s}_{0}} \rightarrow \ldots \mathbf{v}_{\mathbf{s}_{\mathbf{i}}} \rightarrow \mathbf{v}_{\mathbf{s}_{\mathbf{0}}}$, where $\mathbf{v}_{\mathbf{s}_{\mathbf{0}}}, \ldots, \mathbf{v}_{\mathbf{s}_{\mathbf{i}}}$ are distinct.

- Let $\mathcal{V}_{1}$ and $\mathcal{V}_{2}$ represent two subsets, $P$ is a $\mathcal{V}_{1}$-topped path if its end belongs to $\mathcal{V}_{1}$.

Consider now any bipartite graph noted $B$ defined by the triplet $\left(\mathbf{V}^{+}, \mathbf{V}^{-}, W\right)$, and let us recall the subdivision of such graph into $\nu+2$ partially ordered irreducible components denoted $\mathcal{C}_{i}(B)=\left(\mathbf{V}_{\mathbf{i}}^{+}(B), \mathbf{V}_{\mathbf{i}}^{-}(B), W_{i}(B)\right)$ using the Dulmage-Mendelsohn (DM) decomposition (Dulmage and Mendelsohn, 1958; Murota, 1987):

$\leftrightarrow$ Find a maximal matching $M$ in $B$. We associate to this maximal matching a non bipartite digraph noted $B_{M}=$ $\left(\mathbf{V}^{+}, \mathbf{V}^{-}, W_{M}\right)$ where $\left(\mathbf{v}_{\mathbf{1}}, \mathbf{v}_{\mathbf{2}}\right) \in W_{M} \Leftrightarrow\left(\mathbf{v}_{\mathbf{1}}, \mathbf{v}_{\mathbf{2}}\right) \in$ $W$ or $\left(\mathbf{v}_{\mathbf{2}}, \mathbf{v}_{\mathbf{1}}\right) \in M$. We denote by $\partial^{+} \mathbf{M}$ (resp. $\left.\partial^{-} \mathbf{M}\right)$ the set of vertices in $\mathbf{V}^{+}$(resp. in $\mathbf{V}^{-}$) covered by the edges of $M$. We note $\mathbf{S}_{\mathbf{0}}^{+}=\mathbf{V}^{+} \backslash \partial^{+} \mathbf{M}$ and $\mathbf{S}_{\mathbf{0}}^{-}=\mathbf{V}^{-} \backslash \partial^{-} \mathbf{M}$.

$\rightarrow \mathbf{V}_{\mathbf{0}}^{+}(B)=\mathbf{S}_{\mathbf{0}}^{+} \cup\left\{\mathbf{v} \in \mathbf{V}^{+}, \exists\right.$ a path in $B_{M}$ from $\mathbf{S}_{\mathbf{0}}^{+}$to $\left.\mathbf{v}\right\}$. $\rightarrow \mathbf{V}_{\mathbf{0}}^{-}(B)=\left\{\mathbf{v} \in \mathbf{V}^{-}, \exists\right.$ a path in $B_{M}$ from $\mathbf{S}_{\mathbf{0}}^{+}$to $\left.\mathbf{v}\right\}$

$\leftrightarrow W_{0}(B)=\left\{\right.$ edges linking $\mathbf{V}_{\mathbf{0}}^{+}(B)$ to $\left.\mathbf{V}_{\mathbf{0}}^{-}(B)\right\}$.

$\uparrow \mathbf{V}_{\infty}^{+}(B)=\left\{\mathbf{v} \in \mathbf{V}^{+}, \exists\right.$ a path in $B_{M}$ from $\mathbf{v}$ to $\left.\mathbf{S}_{\mathbf{0}}^{-}\right\}$ $\rightarrow \mathbf{V}_{\infty}^{-}(B)=\mathbf{S}_{\mathbf{0}}^{-} \cup\left\{\mathbf{v} \in \mathbf{V}^{-}, \exists\right.$ a path in $B_{M}$ from $\mathbf{v}$ to $\left.\mathbf{S}_{\mathbf{0}}^{-}\right\}$. $\uparrow W_{\infty}(B)=\left\{\right.$ edges linking $\mathbf{V}_{\infty}^{+}(B)$ to $\left.\mathbf{V}_{\infty}^{-}(B)\right\}$.

$\uparrow$ For $i=1, \ldots, \nu$, let $\mathcal{C}_{\mathbf{i}}(B)$ be the strongly connected components ( 2 vertices $\mathbf{v}_{\mathbf{i}}$ and $\mathbf{v}_{\mathbf{j}}$ are said to be strongly connected if it exists path from $\mathbf{v}_{\mathbf{i}}$ to $\mathbf{v}_{\mathbf{j}}$ and a path from $\mathbf{v}_{\mathbf{j}}$ to $\mathbf{v}_{\mathbf{i}}$, the relation "is strongly connected to" is an equivalence relation and we call each equivalent class a strongly connected component) of the graph obtained from $B_{M}$ after deleting the vertices and the edges of $\mathcal{C}_{\mathbf{0}}(B)$ and $\mathcal{C}_{\infty}(B)$. $\uparrow \rightarrow$ Define a partial order " $\preccurlyeq$ " on the strongly connected compo-nents $\mathcal{C}_{\mathbf{i}}(B)$ for $i=0,1, \ldots, \nu, \infty$ as follows: $\mathcal{C}_{\mathbf{i}}(B) \preccurlyeq \mathcal{C}_{\mathbf{j}}(B) \Leftrightarrow$ there exists a path starting from vertices of $\mathcal{C}_{\mathbf{j}}(B)$ to the ones of $\mathcal{C}_{\mathbf{i}}(B)$ in $B_{M}, \mathcal{C}_{\mathbf{0}}(B)$ is called the minimal inconsistent part of $B$ and $\mathcal{C}_{\infty}(B)$ is the maximal inconsistent part of $B$. 


\section{Main result}

\subsection{Preliminaries}

To establish the mode observability of each mode, we apply some results deduced mainly from (Murota, 1987; Boukhobza and Hamelin, 2011). These results allow in a first stage to reduce the graph to its useful part i.e. to the part where it is possible to write a non-trivial expression linking only the output and their derivatives. The DMdecomposition properties allow to prove quite easily the following result:

Lemma 1 For a mode $q$, there can exist a redundancy equation linking the output components and their derivatives only in the maximal inconsistent part $\mathcal{C}_{\infty}\left(B\left(\Sigma_{\Lambda}, q\right)\right)$ of $B\left(\Sigma_{\Lambda}, q\right)$.

According to the previous lemma, we consider in the sequel of the paper only the maximal inconsistent part of $B\left(\Sigma_{\Lambda}, q\right)$. We can then work on bipartite graphs $\bar{B}\left(\Sigma_{\Lambda}, q\right)$ instead of $B\left(\Sigma_{\Lambda}, q\right)$, where $\bar{B}\left(\Sigma_{\Lambda}, q\right)=\left(\overline{\mathbf{V}}_{\mathbf{q}}^{+}, \overline{\mathbf{V}}_{\mathbf{q}}^{-}, \bar{W}_{q}\right)$ with $\overline{\mathbf{V}}_{\mathbf{q}}^{+}=\mathbf{V}_{\infty}^{+}\left(B\left(\Sigma_{\Lambda}, q\right)\right), \overline{\mathbf{V}}_{\mathbf{q}}^{-}=\mathbf{V}_{\infty}^{-}\left(B\left(\Sigma_{\Lambda}, q\right)\right)$ and $\bar{W}_{q}$ is the subset of edges included in $W_{q}$ which link $\mathbf{V}_{\infty}^{+}\left(B\left(\Sigma_{\Lambda}, q\right)\right)$ to $\mathbf{V}_{\infty}^{-}\left(B\left(\Sigma_{\Lambda}, q\right)\right)$. In this framework, $\bar{A}_{q^{-}}$ edges, $\bar{C}_{q}$-edges and $\bar{E}_{q}$-edges are respectively the subsets of $A_{q}$-edges, $C_{q}$-edges and $E_{q}$-edges linking $\mathbf{V}_{\infty}^{+}\left(B\left(\Sigma_{\Lambda}, q\right)\right)$ to $\mathbf{V}_{\infty}^{-}\left(B\left(\Sigma_{\Lambda}, q\right)\right)$. For $q=1,2$, we denote by $\overline{\mathbf{X}}_{\mathbf{q}}, \overline{\mathbf{Y}}_{\mathbf{q}}$ and $\overline{\mathbf{Z}}_{\mathbf{q}}$ the restrictions of respectively $\mathbf{X}, \mathbf{Y}$ and $\mathbf{Z}$ to the vertices of $\overline{\mathbf{V}}_{\mathbf{q}}^{+}$and $\overline{\mathbf{V}}_{\mathbf{q}}^{-}$i.e. $\overline{\mathbf{X}}_{q}=\overline{\mathbf{V}}_{\mathbf{q}}^{+}, \overline{\mathbf{Y}}_{\mathbf{q}}=\overline{\mathbf{V}}_{\mathbf{q}}^{-} \cap \mathbf{Y}$ and $\overline{\mathbf{Z}}_{\mathbf{q}}=\overline{\mathbf{V}}_{\mathbf{q}}^{-} \cap \mathbf{Z}$.

Example 1 (Continued): For both modes 1 and 2, we can choose as maximal matching $M=\left\{\left(\mathbf{x}_{1}, \mathbf{z}_{3}\right),\left(\mathbf{x}_{2}, \mathbf{y}_{2}\right),\left(\mathbf{x}_{3}, \mathbf{y}_{3}\right)\right.$, $\left.\left(\mathbf{x}_{4}, \mathbf{y}_{4}\right),\left(\mathbf{x}_{5}, \mathbf{z}_{4}\right),\left(\mathbf{x}_{\mathbf{6}}, \mathbf{z}_{\mathbf{2}}\right)\right\}$. We can deduce that the maximal inconsistent part is defined by the following set of vertices: $\overline{\mathbf{V}}_{1}^{+}=$ $\overline{\mathbf{V}}_{\mathbf{2}}^{+}=\left\{\mathbf{x}_{1}, \mathbf{x}_{2}, \mathbf{x}_{3}, \mathbf{x}_{4}\right\}$ and $\overline{\mathbf{V}}_{\mathbf{1}}^{-}=\overline{\mathbf{V}}_{\mathbf{2}}^{-}=\left\{\mathbf{y}_{1}, \mathbf{y}_{2}, \mathbf{y}_{\mathbf{3}}, \mathbf{y}_{4}\right.$, $\left.\mathbf{z}_{1}, \mathbf{z}_{3}\right\}$. The reduced bipartite graphs are depicted in Figure 2.
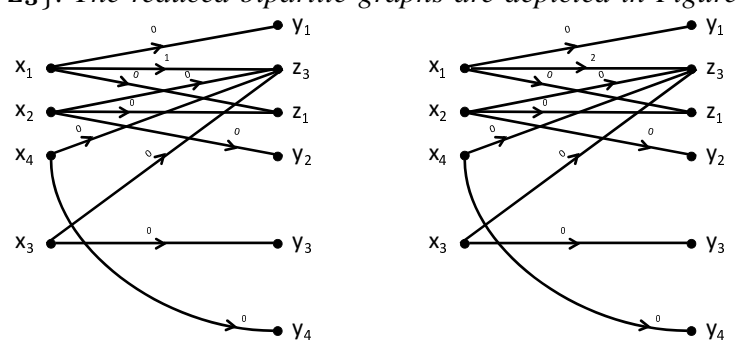

Figure 2. Reduced bipartite graphs of the system of Example 1. Mode 1 on the left, mode 2 on the right.

We define, for each $q=1,2$, and each output vertex subset $\mathbf{V}_{\mathbf{Y}} \subseteq \overline{\mathbf{Y}}_{\mathbf{q}}$, sub-graph $\bar{B}\left(\Sigma_{\Lambda}, q, \mathbf{V}_{\mathbf{Y}}\right)$ by removing from $\bar{B}\left(\Sigma_{\Lambda}, q\right)$ all the output vertices which are not in $\mathbf{V}_{\mathbf{Y}}$ and the $C_{q}$-edges which do not arrive to $\mathbf{V}_{\mathbf{Y}}$. Then, we exhibit two classes of output vertex subsets for which we can compute the observability subspaces dimensions:

- $\mathbf{V}_{\mathbf{Y}} \subseteq \overline{\mathbf{Y}}_{\mathbf{q}}$ is said to be $q$-complete, if the minimal inconsistent part of $\bar{B}\left(\Sigma_{\Lambda}, q, \mathbf{V}_{\mathbf{Y}}\right)$ is empty.

- $\mathbf{V}_{\mathbf{Y}} \subseteq \overline{\mathbf{Y}}_{\mathbf{q}}$ is said to be $q$-eligible, if only the maximal inconsistent part of $\bar{B}\left(\Sigma_{\Lambda}, q, \mathbf{V}_{\mathbf{Y}}\right)$ is not empty.

We can deduce the following results from (Boukhobza and Hamelin, 2011):

Lemma 2 For each q-complete subset $\mathbf{Y}_{\mathbf{u}}=$ $\left\{\mathbf{y}_{\mathbf{i}_{1, \mathbf{q}}}, \mathbf{y}_{\mathbf{i}_{2}}, \ldots, \mathbf{y}_{\mathbf{i}_{\mathbf{k}}}\right\} \subset \overline{\mathbf{Y}}_{\mathbf{q}}$ there exist generically state space subdivision $\bar{X}_{q}=\left(\tilde{X}_{q}^{T}, \hat{X}_{q}^{T}\right)^{T}$, functions $\varphi_{x, q}, \varphi_{y, q}$ and an integer $\nu \leq n$ such that, in mode $q$, dynamics of system (2) restricted to its maximal inconsistent part can be written as:

$$
\begin{aligned}
\tilde{E}_{q} \dot{\tilde{X}}_{q} & =\tilde{A}_{q} \tilde{X}_{q}+\varphi_{x, q}\left(Y_{u}, \dot{Y}_{u}, \ldots, Y_{u}^{(\nu)}\right) \\
Y_{s} & =\tilde{C}_{q} \tilde{X}_{q}+\varphi_{y, q}\left(Y_{u}, \dot{Y}_{u}, \ldots, Y_{u}^{(\nu)}\right)
\end{aligned}
$$

where $\tilde{E}_{q}$ is a square full rank matrix and $\mathbf{Y}_{\mathbf{s}}=\overline{\mathbf{Y}}_{\mathbf{q}} \backslash \mathbf{Y}_{\mathbf{u}}$. Corollary 3 For each $\mathbf{y}_{\mathbf{i}} \in \overline{\mathbf{Y}}_{\mathbf{q}}$, there exist integers $k$ and $s$, and a function $f_{q}$, which satisfy expression on form $y_{i}^{(k)}=$ $f_{q}\left(y_{i}, \dot{y}_{i}, \ldots, y_{i}^{(k-1)}, Y_{u}, \dot{Y}_{u}, \ldots, Y_{u}^{(s)}\right)=0$, where $\mathbf{Y}_{\mathbf{u}} \subseteq \overline{\mathbf{Y}}_{\mathbf{q}} \backslash\left\{\mathbf{y}_{\mathbf{i}}\right\}$. Finally, we characterize the generic dimension of the observability subspace associated to an output vertex subset:

Definition 3 For each mode $q \in\{1,2\}$ and to each $q$ eligible vertex subset $\mathbf{V}_{\mathbf{Y}} \subseteq \overline{\mathbf{Y}}_{\mathbf{q}}$, integer $\beta_{q}\left(\mathbf{V}_{\mathbf{Y}}\right)$ is defined as card $\left(\overline{\mathbf{X}}_{\mathbf{q}}\right)$ minus the sum of minimal number of $\bar{E}_{q}$-edges included in a maximal matching of the maximal inconsistent part of $\bar{B}\left(\Sigma_{\Lambda}, q, \mathbf{V}_{\mathbf{Y}}\right)$. For each $q$-complete vertex subset $\mathbf{V}_{\mathbf{Y}} \subseteq \overline{\mathbf{Y}}_{\mathbf{q}}$, we define $\beta_{q}\left(\mathbf{V}_{\mathbf{Y}}\right)$ as card $\left(\overline{\mathbf{X}}_{\mathbf{q}}\right)$ minus the sum of: - the minimal number of $\bar{E}_{q}$-edges included in a maximal matching of the maximal inconsistent part of $\bar{B}\left(\Sigma_{\Lambda}, q, \mathbf{V}_{\mathbf{Y}}\right)$ - the maximal number of $\bar{E}_{q}$-edges included in a maximal matching of the consistent part of $\bar{B}\left(\Sigma_{\Lambda}, q, \mathbf{V}_{\mathbf{Y}}\right)$.

We can deduce from (Boukhobza and Hamelin, 2011):

Lemma 4 Consider SSDS $\Sigma_{\Lambda}$, for each q-complete vertex subset $\mathbf{V}_{\mathbf{Y}} \subseteq \overline{\mathbf{Y}}_{\mathbf{q}}, \beta_{q}\left(\mathbf{V}_{\mathbf{Y}}\right)$ is equal to the generic dimension of the observable subspace for mode $q$ when restricting the measurements to the output components associated to $\mathbf{V}_{\mathbf{Y}}$.

Definition 4 For each $\mathbf{y}_{\mathbf{i}} \in \overline{\mathbf{Y}}_{\mathbf{q}}$ and mode $q$, we define integer $d_{q}\left(\mathbf{y}_{\mathbf{i}}\right)=\beta_{q}\left(\overline{\mathbf{Y}}_{\mathbf{q}}\right)-\beta_{q}\left(\overline{\mathbf{Y}}_{\mathbf{q}} \backslash\left\{\mathbf{y}_{\mathbf{i}}\right\}\right)$.

\subsection{Discrete mode observability digraph}

The discrete mode observability digraph contains the same vertices than $\bar{B}\left(\Sigma_{\Lambda}, q\right)$ but not the same edges. In fact, we remove all the $\bar{E}_{q}$-edges and substitute them by new edges denoted $\Gamma_{q}$-edges and $\Psi_{q}$-edges defined for each mode $q$.

\section{Definition of $\Gamma_{q}$-edges and their indices:}

$q$ Consider $\bar{B}_{E}\left(\Sigma_{\Lambda}, q\right)$ the restriction of $\bar{B}\left(\Sigma_{\Lambda}, q\right)$ to only the $\bar{E}_{q}$-edges,

$\uparrow$ Let $M$ be a maximal matching in $\bar{B}_{E}\left(\Sigma_{\Lambda}, q\right)$ to which we associate a non bipartite digraph noted $\bar{B}_{q, M}=$ $\left(\overline{\mathbf{X}}_{\mathbf{q}}, \overline{\mathbf{Y}}_{\mathbf{q}} \cup \overline{\mathbf{Z}}_{\mathbf{q}}, W_{M}\right)$ where $\left(\mathbf{v}_{\mathbf{1}}, \mathbf{v}_{\mathbf{2}}\right) \in W_{M} \Leftrightarrow\left(\mathbf{v}_{\mathbf{1}}, \mathbf{v}_{\mathbf{2}}\right) \in$ $\bar{E}_{q}$-edges or $\left(\mathbf{v}_{\mathbf{2}}, \mathbf{v}_{\mathbf{1}}\right) \in M$.

$\leftrightarrow$ In $W_{M}$, if $\left(\mathbf{v}_{\mathbf{1}}, \mathbf{v}_{\mathbf{2}}\right) \in \bar{E}_{q}$-edges, then it conserves its index and if $\left(\mathbf{v}_{\mathbf{1}}, \mathbf{v}_{\mathbf{2}}\right) \in W_{M} \backslash \bar{E}_{q}$-edges, then it has the same index than $\left(\mathbf{v}_{\mathbf{1}}, \mathbf{v}_{\mathbf{2}}\right)$ which belongs to $\bar{E}_{q}$-edges.

$\uparrow$ Let $\mathfrak{E}^{+}(q, 0)$ be the set of vertices in $\overline{\mathbf{X}}_{q}$ which are not covered by the edges of $M$,

$\uparrow$ For each mode $q$, we note by $\mathcal{C}_{i}\left(\bar{E}_{q}\right)=\left(\mathfrak{E}^{+}(q, i)\right.$, $\left.\mathfrak{E}^{-}(q, i), \mathcal{E}(q, i)\right), i=1, \ldots, \mu_{q}$ the strongly connected components related to $\bar{B}_{q, M}$ and $\mathcal{C}_{0}\left(\bar{E}_{q}\right)=\left(\mathfrak{E}^{+}(q, 0), \emptyset, \emptyset\right)$. $\uparrow$ Use the partial order "ঝ" (cf. Section 3 ) on the strongly connected components $\mathcal{C}_{i}\left(\bar{E}_{q}\right)$ for $i=0,1, \ldots, \mu_{q}$.

$\uparrow$ if $\mathcal{C}_{i}\left(\bar{E}_{q}\right)$ contains an edge with index $q$ then $\mathcal{C}_{i}\left(\bar{E}_{q}\right)$ is said to be a $q$-component.

$\leftrightarrow \Gamma_{q}$-edges $=\left\{\left(\mathbf{z}_{\mathbf{j}}, \mathbf{x}_{\mathbf{i}}\right)\right.$ if $\mathbf{x}_{\mathbf{i}}$ and $\mathbf{z}_{\mathbf{j}}$ belong to the same strongly component $\left.\mathcal{C}_{\ell}\left(\bar{E}_{q}\right)\right\} \cup\left\{\left(\mathbf{z}_{\mathbf{j}}, \mathbf{x}_{\mathbf{i}}\right)\right.$ if $\mathbf{x}_{\mathbf{i}} \in \mathfrak{E}^{+}(q, \ell)$, 
$\mathbf{z}_{\mathbf{j}} \in \mathfrak{E}^{-}(q, k)$ and $\left.\mathcal{C}_{\ell}\left(\bar{E}_{q}\right) \preccurlyeq \mathcal{C}_{k}\left(\bar{E}_{q}\right), \ell \neq 0\right\}$.

The indices of the $\Gamma_{q}$-edges are computed as follows: - all the edges linking elements of a $q$-component have in$\operatorname{dex} q$.

- if $\mathcal{C}_{\ell}\left(\bar{E}_{q}\right) \preccurlyeq \mathcal{C}_{k}\left(\bar{E}_{q}\right)$ and if there exists a path in $\bar{B}_{q, M}$ starting from $\mathcal{C}_{\ell}\left(\bar{E}_{q}\right)$ to $\mathcal{C}_{k}\left(\bar{E}_{q}\right)$ containing at least an edge with index $q$, then the $\Gamma_{q}$-edges linking $\mathbf{x}_{\mathbf{i}} \in \mathfrak{E}^{+}(q, \ell)$ to $\mathbf{z}_{\mathbf{j}} \in \mathfrak{E}^{-}(q, k)$ have all index $q$.

- if $\mathcal{C}_{\ell}\left(\bar{E}_{q}\right) \preccurlyeq \mathcal{C}_{k}\left(\bar{E}_{q}\right)$ and if there exists a path starting from $\mathcal{C}_{\ell}\left(\bar{E}_{q}\right)$ to $\mathcal{C}_{k}\left(\bar{E}_{q}\right)$ containing only edges with index 0 , then the $\Gamma_{q}$-edges linking $\mathbf{x}_{\mathbf{i}} \in \mathfrak{E}^{+}(q, \ell)$ to $\mathbf{z}_{\mathbf{j}} \in \mathfrak{E}^{-}(q, k)$ have index 0 .

Definition of $\Psi_{q}$-edges and their indices:

$\uparrow \Psi_{q}$-edges $=\left\{\left(\mathbf{x}_{\mathbf{i}}, \mathbf{z}_{\mathbf{j}}\right)\right.$ if $\mathbf{x}_{\mathbf{i}} \in \mathfrak{E}^{+}(q, 0), \mathbf{z}_{\mathbf{j}} \in \mathfrak{E}^{-}(q, k)$ and $\left.\mathcal{C}_{0}\left(\bar{E}_{q}\right) \preccurlyeq \mathcal{C}_{k}\left(\bar{E}_{q}\right)\right\}$.

The indices of the $\Psi_{q}$-edges are computed as follows:

- if there exists a path in $\bar{B}_{q, M}$ starting from $\mathcal{C}_{0}\left(\bar{E}_{q}\right)$ to $\mathcal{C}_{k}\left(\bar{E}_{q}\right)$ containing at least an edge with index $q$ then the $\Psi_{q^{-}}$ edges linking $\mathbf{x}_{\mathbf{i}} \in \mathfrak{E}^{+}(q, 0)$ to $\mathbf{z}_{\mathbf{j}} \in \mathfrak{E}^{-}(q, k)$ have index $q$. - if there exists a path in $\bar{B}_{q, M}$ starting from $\mathcal{C}_{0}\left(\bar{E}_{q}\right)$ to $\mathcal{C}_{k}\left(\bar{E}_{q}\right)$ containing only edges with index 0 then the $\Psi_{q^{-}}$ edges linking $\mathbf{x}_{\mathbf{i}} \in \mathfrak{E}^{+}(q, 0)$ to $\mathbf{z}_{\mathbf{j}} \in \mathfrak{E}^{-}(q, k)$ have index 0 .

The interpretation of $\Gamma_{q}$-edges and $\Psi_{q}$-edges is related to the decomposition of matrix $\bar{E}_{q}$ into $\left(\tilde{E}_{q} \hat{E}_{q}\right)$, where $\tilde{E}_{q}$ is a square generically full column matrix. Edge subset $\Gamma_{q}$-edges represents matrix $\tilde{E}_{q}^{-1}$ and $\Psi_{q}$-edges represents matrix $\tilde{E}_{q}^{-1} \hat{E}_{q}$ as it is stated in the following lemma proved in Appendix A:

Lemma 5 For $\operatorname{SSDS} \Sigma_{\Lambda}$ in mode $q$, matrix $\bar{E}_{q}$ can be written as $\left(\tilde{E}_{q} \hat{E}_{q}\right)$, after possibly column permutations, where $\tilde{E}_{q}$ is a square generically full column matrix which columns correspond to state vertices included in $\mathfrak{E}^{+}(q, k), k \neq 0$ and $\bar{E}_{q}$ corresponds to state vertices included in $\mathfrak{E}^{+}(q, 0)$. We can state:

$i$. The $\Gamma_{q}$-edges correspond generically to the edges of $\tilde{E}_{q}^{-1}$ and when the index of an edge of $\Gamma_{q}$-edges is equal to $q$, then the corresponding element of $\tilde{E}_{q}^{-1}$ is specific to mode $q$.

ii. The $\Psi_{q}$-edges correspond generically to the edges of $\tilde{E}_{q}^{-1} \hat{E}_{q}$ and when the index of an edge of $\Psi_{q}$-edges is equal to $q$, then the corresponding element of $\tilde{E}_{q}^{-1} \hat{E}_{q}$ is specific to mode $q$.

Using the previous $\Gamma_{q}$ and $\Psi_{q}$ edges definitions, we construct, for each mode $q=1,2$, a new digraph noted $\mathcal{G}_{d m o}\left(\Sigma_{\Lambda}, q\right)$ defined by $\left(\overline{\mathbf{V}}_{\mathbf{q}}^{+}, \overline{\mathbf{V}}_{\mathbf{q}}^{-}, \bar{W}_{q} \cup \Gamma_{q}\right.$-edges $\cup$ $\Psi_{q}$-edges $\backslash \bar{E}_{q}$-edges $)$, where each edge belonging to $\bar{A}_{q} \cup \bar{C}_{q}$ keeps its initial index.

Example 1 (Continued): For mode 1 , to cover $\overline{\mathbf{Z}}_{1}$ with only $\bar{E}_{1}$-edges, we choose the maximal matching $M=$ $\left\{\left(\mathbf{x}_{1}, \mathbf{z}_{1}\right),\left(\mathbf{x}_{2}, \mathbf{z}_{3}\right)\right\}$. Therefore, $\mathfrak{E}^{+}(1,0)=\left\{\mathbf{x}_{3}, \mathbf{x}_{4}\right\}$ which vertices are not covered by $M$. When we carry out the decomposition of $\bar{B}_{M}\left(\Sigma_{\Lambda}, 1\right)$ into strongly connected components, we obtain two strongly connected components: $\mathcal{C}_{1}\left(\bar{E}_{1}\right)$ defined by $\mathfrak{E}^{+}(1,1)=\left\{\mathbf{x}_{\mathbf{2}}\right\}$ and $\mathfrak{E}^{-}(1,1)=\left\{\mathbf{z}_{\mathbf{3}}\right\}$ and $\mathcal{C}_{2}\left(\bar{E}_{1}\right)$ defined by $\mathfrak{E}^{+}(1,2)=\left\{\mathbf{x}_{1}\right\}$ and $\mathfrak{E}^{-}(1,2)=\left\{\mathbf{z}_{1}\right\}$, with $\mathcal{C}_{1}\left(\bar{E}_{1}\right) \npreceq \mathcal{C}_{2}\left(\bar{E}_{1}\right)$ and $\mathcal{C}_{2}\left(\bar{E}_{1}\right) \npreceq \mathcal{C}_{1}\left(\bar{E}_{1}\right)$. These two strongly connected components are not 1-components. We can then deduce that there exist two $\Gamma_{1}$ edges with indices $0:\left\{\left(\mathbf{z}_{1}, \mathbf{x}_{\mathbf{1}}\right),\left(\mathbf{z}_{\mathbf{3}}, \mathbf{x}_{\mathbf{2}}\right)\right\}$. Finally, as there exists a path between $\mathfrak{E}^{+}(1,0)$ and $\mathcal{C}_{1}\left(\bar{E}_{1}\right)\left(\mathbf{x}_{3} \rightarrow \mathbf{z}_{3}\right)$, which does not contain any edge with index 1 , we have one $\Psi_{1}$-edge having index $0:\left(\mathbf{x}_{3}, \mathbf{z}_{3}\right)$. The discrete mode observability digraph associated to mode 1 is depicted in the left side of Figure 3. Similarly, the

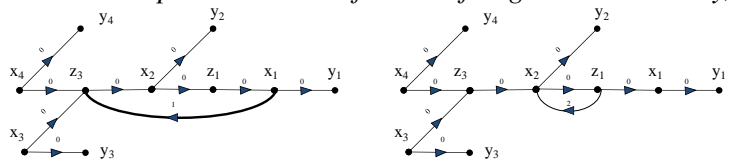

Figure 3. Discrete mode observability digraphs for the system of Example 1. Mode 1 (left), Mode 2 (right).

discrete mode observability digraph for mode 2 is depicted in the right side of Figure 3.

In the new digraph, we define two particular vertex subsets: - For $q \in\{1,2\}, V_{e s s, q}\left[\mathcal{V}_{1}, \mathcal{V}_{2}\right]$ is the vertex subset including the vertices present in all the maximum $\mathcal{V}_{1}-\mathcal{V}_{2}$ linkings included in $\mathcal{G}_{d m o}\left(\Sigma_{\Lambda}, q\right)$.

- For $q \in\{1,2\}$, in $\mathcal{G}_{d m o}\left(\Sigma_{\Lambda}, q\right)$, there exists a unique vertex subset noted $S_{q}^{o}\left[\mathcal{V}_{1}, \mathcal{V}_{2}\right]$, called minimum output separator, which is the set of begin vertices of all direct $V_{e s s, q}\left[\mathcal{V}_{1}, \mathcal{V}_{2}\right]-\mathcal{V}_{2}$ paths. Note that, an output vertex subset $\mathbf{V}_{\mathbf{Y}}$ is $q$-eligible if in $\mathcal{G}_{d m o}\left(\Sigma_{\Lambda}, q\right), S_{q}^{o}\left[\mathfrak{E}^{+}(q, 0), \mathbf{V}_{\mathbf{Y}}\right]=$ $\mathfrak{E}^{+}(q, 0)$.

\subsection{Discrete mode observability analysis}

Proposition $6 \operatorname{SSDS} \Sigma_{\Lambda}$, with two possible modes $q \in$ $\{1,2\}$ is generically discrete mode observable iff one of the following conditions is satisfied in its associated discrete mode observability digraphs $\mathcal{G}_{\text {dmo }}\left(\Sigma_{\Lambda}, q\right), q=1,2$ :

1. for some $q \in\{1,2\}$, there exists a cycle $\mathcal{C}$ containing at least one edge of index $q$;

2. for some $q \in\{1,2\}$, there exists a $\mathfrak{E}^{+}(q, 0)-\overline{\mathbf{Y}}_{\mathbf{q}}$ path containing at least one edge of index $q$;

3. for $q \neq q^{\prime}, \overline{\mathbf{Y}}_{\mathbf{q}} \neq \overline{\mathbf{Y}}_{\mathbf{q}^{\prime}}$;

4. for some $q \in\{1,2\}$, there exists a q-eligible vertex subset $\mathbf{V}_{\mathbf{Y}} \subseteq \overline{\mathbf{Y}}_{\mathbf{q}}$ such that, either $\mathbf{V}_{\mathbf{Y}}$ is not q-eligible for mode $q^{\prime}$, or $\beta_{q}\left(\mathbf{V}_{\mathbf{Y}}\right) \neq \beta_{q^{\prime}}\left(\mathbf{V}_{\mathbf{Y}}\right)$;

5. for some $q \in\{1,2\}$, there exist an edge $e_{\kappa}$ of index $q$ and an $\mathbf{y}_{\mathbf{i}}$-topped path $P$ of length strictly greater or equal to $2 d_{q}\left(\mathbf{y}_{\mathbf{i}}\right)+1$ which covers $e_{\kappa}$ and such that $\mathbf{v}_{\ell}$ belongs to a direct $S_{q}^{o}\left[\left\{\mathbf{v}_{\mathbf{P}}\right\}, \overline{\mathbf{Y}}_{\mathbf{q}}\right]-\mathbf{y}_{\mathbf{i}}$ path, where $\mathbf{v}_{\mathbf{P}}$ and $\mathbf{v}_{\ell}$ are respectively the begin vertex of $P$ and the end vertex of $e_{\kappa}$.

Proof: Sufficiency: Condition 1: Let us denote by $\mathrm{x}_{\mathbf{i}}$ one of the vertices of cycle $\mathcal{C}$ verifying Condition 1. There exist a $\mathbf{x}_{\mathbf{i}}-\mathbf{y}_{\mathbf{j}}$ path in $\mathcal{G}_{d m o}\left(\Sigma_{\Lambda}, q\right)$, where $\mathbf{y}_{\mathbf{j}} \in \overline{\mathbf{Y}}_{\mathbf{q}}$ and a q-complete subset $\mathbf{Y}_{\mathbf{u}} \subset \overline{\mathbf{Y}}_{\mathbf{q}} \backslash\left\{\mathbf{y}_{\mathbf{j}}\right\}$. From Lemma 2, there exist functions $\varphi_{x, q}$, $\varphi_{y, q}$ and an integer $\nu \geq 0$ such that for mode $q, \Sigma_{\Lambda}$ restricted to its maximal inconsistent part can be written as in form (3). The digraph representation of (3) contains also $\mathcal{C}$. Note that $\mathcal{C}$ is necessarily a succession of $\tilde{E}_{q}^{-1}$ and $\tilde{A}_{q}$ edges. From Theorem 21.1 of (Reinschke, 1988), the characteristic equation of matrix $\tilde{E}_{q}^{-1} \tilde{A}_{q}$, which has the form $\left(\tilde{E}_{q}^{-1} \tilde{A}_{q}\right)^{\tilde{n}}+\ldots+a_{k}\left(\tilde{E}_{q}^{-1} \tilde{A}_{q}\right)^{k}+\ldots$ $+a_{0} I=0$, contains a term $a_{\tilde{n}-\bar{k}}\left(\tilde{E}_{q}^{-1} \tilde{A}_{q}\right)^{\tilde{n}-\bar{k}}$, where $2 \bar{k}$ is the length of $\mathcal{C}$ and $\tilde{n}$ is the dimension of $\tilde{X}_{q} \cdot a_{\tilde{n}-\bar{k}}$ depends on the weight of $\mathcal{C}$ and so, on a specific parameter of $E_{q}^{s}$ or $A_{q}^{s}$. Thus, $\left(\widetilde{C}_{q, j}\left(\tilde{E}_{q}^{-1} \tilde{A}_{q}\right)^{\tilde{n}}+\ldots+a_{k} \tilde{C}_{q, j}\left(\tilde{E}_{q}^{-1} \tilde{A}_{q}\right)^{k}+\ldots+a_{0} \tilde{C}_{q, j}\right) \tilde{X}_{q}=0$ where $\tilde{C}_{q, j}$ is the line of matrix $\widetilde{C}$ related to output $y_{j}$ in mode $q$ in (3) i.e. $y_{j}=\tilde{C}_{q, j} \tilde{X}_{q}+\varphi_{y, q, j}\left(Y_{u}, \dot{Y}_{u}, \ldots, Y_{u}^{(\nu)}\right)$. Equation 
(4) leads to an algebraic equation parametrized by at least an element specific to mode q.

Condition 2: Let denote by $\mathbf{v}_{\mathbf{i}}$ the vertex of $\mathfrak{E}^{+}(q, 0)$ from which there is a $\mathfrak{E}^{+}(q, 0)-\overline{\mathbf{Y}}_{\mathbf{q}}$ path $P$ containing at least an edge $e_{\kappa}$ of index $q$. Let $\lambda_{\kappa}$ be the non-zero parameter (or weight) associated to $e_{\kappa}, \mathbf{y}_{\mathbf{j}}$ be the end of $P$ and $2 \ell+1$ its length. It exists $a$ q-complete subset $\mathbf{Y}_{\mathbf{u}}=\left\{\mathbf{y}_{\mathbf{i}_{1}}, \mathbf{y}_{\mathbf{i}_{2}}, \ldots, \mathbf{y}_{\mathbf{i}_{\mathbf{k}}}\right\} \subset \overline{\mathbf{Y}}_{\mathbf{q}} \backslash\left\{\mathbf{y}_{\mathbf{j}}\right\}$ such that elements of $\mathfrak{E}^{+}(q, 0)$ are covered by a complete matching in $\bar{B}\left(\Sigma_{\Lambda}, q\right)$ without using edges and vertices of $P$ neither the ones belonging to $\bar{E}_{q}$-edges. From Lemma 2 , there exist functions $\varphi_{x, q}, \varphi_{y, q}$ and an integer $\nu \leq \tilde{n}$ such that the dynamics equation of state part $\tilde{X}_{q}$ is in form (3). From the characteristic equation of matrix $\tilde{A}_{q}$, we can write an equation as (4), where also $\tilde{C}_{q, j}$ is the line of matrix $\widetilde{C}$ related to output $y_{j}$ in mode $q$ in (3) i.e. $y_{j}=\tilde{C}_{q, j} X_{1, q}+\varphi_{y, q, j}\left(Y_{u}, \dot{Y}_{u}, \ldots, Y_{u}^{(\nu)}\right)$. Thus, $\tilde{C}_{q, j}\left(\tilde{E}_{q}^{-1} \tilde{A}_{q}\right)^{k} \tilde{X}_{q}=y_{j}^{(k)}-\tilde{C}_{q, j}\left(\varphi_{x, q}^{(k-1)}\left(Y_{u}, \dot{Y}_{u}, \ldots, Y_{u}{ }^{(\nu)}\right)+\right.$ $\tilde{E}_{q}^{-1} \tilde{A}_{q} \varphi_{x, q}^{(k-2)}\left(Y_{u}, \dot{Y}_{u}, \ldots, Y_{u}{ }^{(\nu)}\right)+\ldots+\left(\tilde{E}_{q}^{-1} \tilde{A}_{q}\right)^{k-2}$ $\left.\dot{\varphi}_{x, q}\left(Y_{u}, \dot{Y}_{u}, \ldots, Y_{u}^{(\nu)}\right)\right)-\varphi_{y, q, j}^{(k)}\left(Y_{u}, \dot{Y}_{u}, \ldots, Y_{u}^{(\nu)}\right)$. Since there exists a path of length $2 \ell+1$ from $\mathbf{v}_{\mathbf{i}}$ to $\mathbf{y}_{\mathbf{j}}$ containing an edge $e_{\kappa}$, term $C_{j}\left(\tilde{E}_{q}^{-1} \tilde{A}_{q}\right)^{\ell}$ is not zero and depends on the element denoted previously $\lambda_{\kappa}$. Therefore, we obtain an algebraic equation specific to mode $q$.

Condition 3: This condition implies that there exists $\mathbf{y}_{\mathbf{i}} \in \overline{\mathbf{Y}}_{\mathbf{q}} \backslash \overline{\mathbf{Y}}_{\mathbf{q}^{\prime}}$. Using Corollary 3 , there is a redundancy equation including $y_{i}$ and its derivatives in mode q but not in mode $q^{\prime}$. Condition 4: If $\mathbf{V}_{\mathbf{Y}}$ is q-eligible for mode $q$ and not for mode $q^{\prime}$, then Corollary 3 ensures the existence of an equation linking the components of $\mathbf{V}_{\mathbf{Y}}$ and their derivatives in mode $q$ but not in mode $q^{\prime}$. Otherwise, if $\beta_{q}\left(\mathbf{V}_{\mathbf{Y}}\right) \neq \beta_{q^{\prime}}\left(\mathbf{V}_{\mathbf{Y}}\right)$, then at least one output derivative can be expressed in mode $q^{\prime}$ using other outputs but not in mode $q$.

Condition 5: If, for some $q, \mathbf{y}_{\mathbf{i}} \in \overline{\mathbf{Y}}_{\mathbf{q}}$ then there exists a $q$ complete vertex subset $\mathbf{Y}_{\mathbf{u}} \subseteq \overline{\mathbf{Y}}_{\mathbf{q}} \backslash\left\{\mathbf{y}_{\mathbf{i}}\right\}$. From Lemma 2, there exist functions $\varphi_{x, q}, \varphi_{y, q}$ and an integer $\nu \leq \tilde{n}$ such that the dynamics equation of state part $\tilde{X}_{q}$ is in form (3). Moreover, there exists a minimal subset $\mathbf{Y}_{\mathbf{s}} \subseteq \overline{\mathbf{Y}}_{\mathbf{q}} \backslash\left(\mathbf{Y}_{\mathbf{u}} \cup\left\{\mathbf{y}_{\mathbf{i}}\right\}\right)$ such that $\forall k \geq d_{q}\left(\mathbf{y}_{\mathbf{i}}\right)$, we can write an equation of the form

$$
y_{i}^{(k)}=\sum_{s<\tilde{k_{i}}} \alpha_{i, s} y_{i}^{(s)}+\sum_{l \mid \mathbf{y}_{l} \in \mathbf{Y}_{\mathbf{s}}} \sum_{s=0}^{\tilde{n}} \alpha_{l, s} y_{l}^{(s)}+v\left(Y_{u}, \ldots, Y_{u}^{(n)}\right)
$$

Let us denote by $\mathbf{v}_{\mathbf{P}}=\mathbf{x}_{\mathbf{j}}$ the begin vertex of the so-called path $P$ satisfying Condition $\mathbf{5}$ (i.e. $P$ is a $\mathbf{y}_{\mathbf{i}}$-topped path of length $2 k+1$ strictly greater than $d_{q}\left(\mathbf{y}_{\mathbf{i}}\right)$ and covers $\left.e_{\kappa}\right)$ and $\mathrm{e}_{j}$ the $j^{\text {th }}$ Euclidean vector. Relation (5) can be written as:

$$
\begin{aligned}
& \tilde{C}_{q, i}\left(\tilde{E}_{q}^{-1} \widetilde{A}_{q}\right)^{k} \mathrm{e}_{j}= \\
& \left(\sum_{s<d_{q}\left(\mathbf{y}_{\mathbf{i}}\right)} \alpha_{i, s} \tilde{C}_{q, i}\left(\tilde{E}_{q}^{-1} \widetilde{A}_{q}\right)^{s}+\sum_{\mathbf{y}_{l} \in \mathbf{Y}_{\mathbf{s}}} \sum_{s=0}^{n} \alpha_{l, s} \tilde{C}_{q, l}\left(\tilde{E}_{q}^{-1} \widetilde{A}_{q}\right)^{s}\right) \mathrm{e}_{j}
\end{aligned}
$$

where each non-zero component of $\tilde{C}_{q, l}\left(\tilde{E}_{q}^{-1} \widetilde{A}_{q}\right)^{s}$ is associated to the paths arriving to $\mathbf{y}_{l} \in \mathbf{Y}_{\mathbf{s}}$ of length $2 s+1$. If $S_{q}^{o}\left[\left\{\mathbf{x}_{\mathbf{j}}\right\}, \mathbf{Y}_{\mathbf{s}} \cup\left\{\mathbf{y}_{\mathbf{i}}\right\}\right]$ is a state vertex, let us denote it $\mathbf{x}_{\mathbf{r}}$. There exist $k_{r}$ and $k^{\prime}$ such that $k_{r}+k^{\prime}=k$ and $\tilde{C}_{q, i}\left(\tilde{E}_{q}^{-1} \widetilde{A}_{q}\right)^{k} \mathrm{e}_{j}=\tilde{C}_{q, i}\left(\tilde{E}_{q}^{-1} \widetilde{A}_{q}\right)^{k_{r}} \Delta_{r}\left(\tilde{E}_{q}^{-1} \widetilde{A}_{q}\right)^{k^{\prime}} \mathrm{e}_{j}$ where $\Delta_{r}$ is a diagonal matrix which has only one non-zero element $\Delta_{r}(r, r)=1$. We can do the same reasoning for each term $\tilde{C}_{q, l}\left(\tilde{E}_{q}^{-1} \widetilde{A}_{q}\right)^{s} \mathrm{e}_{j}$ and so there exist $s_{r}$ and $s^{\prime}$ such that $s_{r}+s^{\prime}=s$ and $\tilde{C}_{q, l}\left(\tilde{E}_{q}^{-1} \widetilde{A}_{q}\right)^{s} \mathrm{e}_{j}=\tilde{C}_{q, l}\left(\tilde{E}_{q}^{-1} \widetilde{A}_{q}\right)^{s_{r}} \Delta_{r}\left(\tilde{E}_{q}^{-1} \widetilde{A}_{q}\right)^{s^{\prime}} \mathrm{e}_{j}$. The fact that end vertex $\mathbf{x}_{\ell}$ of $e_{\kappa}$ belongs to a direct $S_{q}^{o}\left[\left\{\mathbf{x}_{\mathbf{j}}\right\}, \overline{\mathbf{Y}}_{\mathbf{q}}\right]-\mathbf{y}_{\mathbf{i}}$ path implies that specific edge $e_{\kappa}$ belongs to a $S_{q}^{o}\left[\mathbf{v}_{\mathbf{P}}, \mathbf{Y}_{\mathbf{s}} \cup\left\{\mathbf{y}_{\mathbf{i}}\right\}\right]-\mathbf{Y}_{\mathbf{s}} \cup\left\{\mathbf{y}_{\mathbf{i}}\right\}$ path. This means that edge $e_{\kappa}$ appears in only some $S_{q}^{o}\left[\mathbf{v}_{\mathbf{P}}, \mathbf{Y}_{\mathbf{s}} \cup\left\{\mathbf{y}_{\mathbf{i}}\right\}\right]-\mathbf{Y}_{\mathbf{s}} \cup\left\{\mathbf{y}_{\mathbf{i}}\right\}$ paths. Thus, some terms of $\tilde{C}_{q, i}\left(\tilde{E}_{q}^{-1} \widetilde{A}_{q}\right)^{k_{r}}$ and $\tilde{C}_{q, l}\left(\tilde{E}_{q}^{-1} \widetilde{A}_{q}\right)^{s_{r}}$, but not all, contain the non-zero parameter corresponding specific to mode q. Denoting by $C_{r}=\mathrm{e}_{r}^{T}$, where $\mathrm{e}_{r}$ is the $r^{\text {th }}$ Euclidean vector, we have that $\tilde{C}_{q, i}\left(\tilde{E}_{q}^{-1} \tilde{A}_{q}\right)^{k} \mathrm{e}_{j}=$ $\tilde{C}_{q, i}\left(\tilde{E}_{q}^{-1} \tilde{A}_{q}\right)^{k_{r}} \Delta_{r}\left(\tilde{E}_{q}^{-1} \widetilde{A}_{q}\right)^{k^{\prime}} \mathrm{e}_{j}=\alpha^{\prime} C_{r}\left(\tilde{E}_{q}^{-1} \widetilde{A}_{q}\right)^{k^{\prime}} \mathrm{e}_{j}$ and $\tilde{C}_{q, l}\left(\tilde{E}_{q}^{-1} \widetilde{A}_{q}\right)^{s} \mathrm{e}_{j}=\tilde{C}_{q, l}\left(\tilde{E}_{q}^{-1} \widetilde{A}_{q}\right)^{s_{r}} \Delta_{r}\left(\tilde{E}_{q}^{-1} \widetilde{A}_{q}\right)^{s^{\prime}} \mathrm{e}_{j}=$ $\alpha_{l, s}^{\prime} C_{r}\left(\tilde{E}_{q}^{-1} \widetilde{A}_{q}\right)^{s^{\prime}} \mathrm{e}_{j}$. Thus, after substitution of the previous terms in relation (6),

$\left(_{s_{r} \leq s<d_{q}\left(\mathbf{y}_{\mathbf{i}}\right)}^{\alpha^{\prime} C_{r}\left(\tilde{E}_{q}^{-1} \widetilde{A}_{q}\right)^{k^{\prime}} \mathrm{e}_{j}=} \alpha_{l \mid \mathbf{y}_{l} \in \mathbf{Y}_{\mathbf{s}}} \sum_{s=s_{r}}^{\tilde{n}} \alpha_{l, s}^{\prime} \alpha_{l, s} C_{r}\left(\tilde{E}_{q}^{-1} \widetilde{A}_{q}\right)^{s-s_{r}}\right) \mathrm{e}_{j}$

where some, but not all, coefficients $\alpha^{\prime}$ and $\alpha_{l, s}^{\prime}$ depend on the weight of $e_{\kappa}$. This equality leads to an algebraic relation satisfied only in mode $q$. If $S_{q}^{o}\left[\left\{\mathbf{x}_{\mathbf{j}}\right\}, \mathbf{Y}_{\mathbf{s}} \cup\left\{\mathbf{y}_{\mathbf{i}}\right\}\right]$ is a dynamical vertex, we have the same result.

Necessity: From Lemma 1, only the edges in the maximal in-

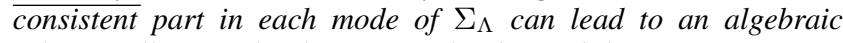
relation allowing the discrete mode observability. It is necessary to consider a q-complete subset $\mathbf{Y}_{\mathbf{u}} \subseteq \overline{\mathbf{Y}}_{\mathbf{q}}$ to obtain for mode $q$ a system on form (3).

When Conditions 1 and 2 are not satisfied, it is not possible, for any choice of $\mathbf{Y}_{\mathbf{u}}$, to obtain an algebraic relation achieving mode distinguishability from the characteristic equation of any matrix $\left(\tilde{E}_{q}^{-1} \widetilde{A}_{q}\right)$ of (3). In this case also, $\varphi_{x, q}\left(Y_{u}, \dot{Y}_{u}, \ldots, Y_{u}^{(\nu)}\right)$ does not depend on any specific element of $\bar{A}_{q}, \bar{C}_{q}$ or $\bar{E}_{q}, q \in\{1,2\}$. When Conditions $\mathbf{3}$ and $\mathbf{4}$ are not satisfied, the output elements of some vertex subset $\mathbf{Y}_{\mathbf{s}}$ implicated in any of the existing algebraic output equations have similar derivative degrees for modes 1 and 2. Thus, the only way to have different algebraic equations is that there exists at least one coefficient $\alpha_{i, s}$ or $\alpha_{l, s}$ of (5), which characterizes a parameter which intervenes in only one mode. Without loss of generality, we can assume that $\mathbf{Y}_{\mathbf{s}}$ is minimal. Defining $\widetilde{k}_{i}=\beta_{q}\left(\mathbf{Y}_{\mathbf{s}} \cup\left\{\mathbf{y}_{\mathbf{i}}\right\} \cup \mathbf{Y}_{\mathbf{u}}\right)-\beta_{q}\left(\mathbf{Y}_{\mathbf{s}} \cup \mathbf{Y}_{\mathbf{u}}\right) \geq d_{q}\left(\mathbf{y}_{\mathbf{i}}\right)$, in redundancy relation (5), we have necessarily $k \geq \widetilde{k_{i}}$. To guarantee discrete mode observability, there must exist at least a $\mathbf{y}_{\mathbf{i}}$-topped path $P$, of length greater or equal to $2 \widetilde{k_{i}}+1$ and so of length strictly greater than $2 d_{q}\left(\mathbf{y}_{\mathbf{i}}\right)$, associated to this relation. Let us denote by $\mathbf{x}_{\mathbf{j}}$ the begin vertex of $P$ and $\mathrm{e}_{j}$ the $j^{\text {th }} E u$ clidean vector. Equation (5) can be written as relation (6), where $\tilde{C}_{q, l}\left(\tilde{E}_{q}^{-1} \widetilde{A}_{q}\right)^{s}$ is associated with $\mathbf{y}_{l}$-topped paths, $\mathbf{y}_{l} \in \mathbf{Y}_{\mathbf{s}}$ of length $2 s+1$. If for each $\mathbf{x}_{\ell}$, end vertex of a specific edge belonging to $P, \mathbf{x}_{\ell}$ does not belong to any direct $S_{q}^{o}\left[\left\{\mathbf{v}_{\mathbf{P}}\right\}, \mathbf{Y}_{\mathbf{1}, \mathbf{q}}\right]-\mathbf{y}_{\mathbf{i}}$ path, then all the paths starting from $\mathbf{x}_{\mathbf{r}}$ to $\mathbf{Y}$ contain only edges common to the two modes. Then all the existing relations of the form (5) do not contain terms specific to a mode q. We can do the same reasoning for all $q$-complete subsets $\mathbf{Y}_{\mathbf{u}} \subseteq \overline{\mathbf{Y}}_{\mathbf{q}}$. Thus, it is not possible to achieve the mode distinguishability. $\triangle$

Comments and interpretation: If there is a specific edge belonging to any cycle in the discrete mode observability digraphs $\mathcal{G}_{d m o}\left(\Sigma_{\Lambda}, q\right)$ then the distinguishability is possible (first condition). If a specific edge belongs to any path linking the minimal inconsistent part of $E_{q}$ to the output in $\mathcal{G}_{d m o}\left(\Sigma_{\Lambda}, q\right)$ then the distinguishability is possible (second condition). If a specific edge allows to modify the output subdivision due to the previous DM-decomposition (third condition) or to modify the observability subspace of any output measurements set (fourth condition), then the distinguishability is possible. Finally, if a specific edge belongs to an output rooted path including a state vertex, which can be linked independently to other outputs, then the distinguishability is possible (fifth condition). If all the conditions are not satisfied, then the two modes are too similar or their differences are not observable from the 
measurements and so they are not distinguishable.

Example 1 (Continued): For mode 1, we have only one specific edge $\left(\mathbf{x}_{1}, \mathbf{z}_{3}\right)$ belonging to a cycle: $\mathbf{x}_{1} \rightarrow \mathbf{z}_{3} \rightarrow \mathbf{x}_{\mathbf{2}} \rightarrow \mathbf{z}_{1} \rightarrow \mathbf{x}_{1}$ and Condition 1 is satisfied. This characterizes an algebraic relation specific to mode 1: $\lambda_{9} \lambda_{11} \ddot{y}_{1}=\lambda_{1} \lambda_{5}\left(\frac{\lambda_{3}}{\lambda_{8}} y_{4}+\frac{\lambda_{14}}{\lambda_{5}} y_{1}-\frac{\lambda_{12}}{\lambda_{7}} \dot{y}_{3}\right)$. For mode 2 , we have only one specific edge $\left(\mathbf{z}_{1}, \mathbf{x}_{2}\right)$ belonging to a cycle: $\mathbf{z}_{1} \rightarrow \mathbf{x}_{2} \rightarrow \mathbf{z}_{1}$. This characterizes an algebraic relation specific to mode 2: $\lambda_{9} \lambda_{11} \ddot{y}_{1}=\lambda_{1} \lambda_{5}\left(\frac{\lambda_{3}}{\lambda_{8}} y_{4}-\frac{\lambda_{16}}{\lambda_{5}} \dot{y}_{1}-\frac{\lambda_{12}}{\lambda_{7}} \dot{y}_{3}\right)$. Condition 2 is not satisfied for mode 1 neither in mode 2 because the direct $\mathfrak{E}^{+}(1,0)-\overline{\mathbf{Y}}_{\mathbf{1}}$ paths do not contain specific edge. Condition $\mathbf{3}$ is not satisfied also as $\overline{\mathbf{Y}}_{\mathbf{1}}=\overline{\mathbf{Y}}_{\mathbf{2}}$. Condition 4 is satisfied for $\mathbf{V}_{\mathbf{y}}=\left\{\mathbf{y}_{\mathbf{2}}, \mathbf{y}_{\mathbf{3}}, \mathbf{y}_{\mathbf{4}}\right\}$ which is 1-eligible and 2eligible and $\beta_{1}\left(\mathbf{V}_{\mathbf{y}}\right)=4$ while $\beta_{2}\left(\mathbf{V}_{\mathbf{y}}\right)=3$. This characterizes an algebraic relation specific to mode 2 and not satisfied for mode 1: $\frac{\lambda_{11}}{\lambda_{6}} \dot{y}_{2}+\frac{\lambda_{12}}{\lambda_{7}} \dot{y}_{3}+\frac{\lambda_{16} \lambda_{1}}{\lambda_{9} \lambda_{6}} y_{2}=\frac{\lambda_{3}}{\lambda_{8}} y_{4}$. For Condition 5, only $\mathbf{y}_{\mathbf{1}}$ or $\mathbf{y}_{\mathbf{2}}$ can play the role of $\mathbf{y}_{\mathbf{i}}$ in Proposition 6 because they are successor of $\mathbf{x}_{1} \cdot d_{1}\left(\mathbf{y}_{1}\right)=0=d_{1}\left(\mathbf{y}_{2}\right)$ and $S_{1}^{o}\left[\left\{\mathbf{x}_{\mathbf{1}}\right\}, \overline{\mathbf{Y}}_{\mathbf{1}}\right]=\left\{\mathbf{x}_{\mathbf{1}}\right\}$. The existence of direct path $\mathbf{x}_{\mathbf{1}}-\mathbf{y}_{\mathbf{2}}$ of length 3 covering specific edge $\left(\mathbf{x}_{1}, \mathbf{z}_{3}\right)$ allows to satisfy Condition 5. This characterizes another algebraic relation specific to mode 1: $\frac{\lambda_{11}}{\lambda_{6}} \dot{y}_{2}+\frac{\lambda_{12}}{\lambda_{7}} \dot{y}_{3}=\frac{\lambda_{3}}{\lambda_{8}} y_{4}+\frac{\lambda_{14}}{\lambda_{5}} y_{1}$. For mode 2, Condition 5 is satisfied also and we have relation: $\frac{\lambda_{11}}{\lambda_{6}} \dot{y}_{2}+\frac{\lambda_{12}}{\lambda_{7}} \dot{y}_{3}+\frac{\lambda_{16}}{\lambda_{5}} \dot{y}_{1}=\frac{\lambda_{3}}{\lambda_{8}} y_{4}$.

\section{Conclusion}

In this paper, we propose a graph-theoretic tool to characterize exactly the generic discrete mode observability of structured switching descriptor linear systems. The studied system can be under-determined, overdetermined or square and possibly non-regular. Our approach consists in two steps. First, we simplify the bipartite graphs associated to the system and then we build a new digraph dedicated to the discrete mode observability. Then, we establish the necessary and sufficient graphical conditions for the discrete mode observability. These conditions generalize the ones established in (Boukhobza and Hamelin, 2011) for systems in standard form. To check these conditions, we can use classical programming techniques, which are free from numerical difficulties as their computational complexity is polynomial.

\section{References}

Babaali, M. \& Pappas, G. J., (2005). Observability of switched linear systems in continuous time. In M. Morari and L. Thiele, Editors, Hybrid Systems: Computation and Control, volume 3414 of Lecture Notes in Computer Science, 103-117. Springer Berlin / Heidelberg.

Boukas, E. (2008). Control of Singular Systems with Random Abrupt Changes.Communications and Control Engineering. Springer-Verlag, Berlin, Heidelberg.

Boukhobza, T., \& Hamelin, F. (2011b). Observability analysis and sensor location study for structured linear systems in descriptor form with unknown inputs. Automatica, 47(12), 2678-2683.

Boukhobza, T., \& Hamelin, F. (2011). Observability of switching structured linear systems with unknown input: a graph-theoretic approach. Automatica, 47(2), 395-402.

Clotet, J., Ferer, J. \& Magret, M. D. (2009). Switched singular linear systems. Proceedings of the $17^{\text {th }}$ Mediterranean Conference on Control and Automation, Greece.

De Koning, W.L.(2003). Digital optimal reduced-order control of pulse-width modulated switched linear systems. Automatica, 39(11), 1997-2003.

Dion, J-M., Commault, C., \& Van der Woude, J. (2003). Generic properties and control of linear structured systems: A survey. Automatica, 39(7), 1125-1144.

Dulmage, A. L. \& Mendelsohn, N. S. (1958). Coverings of bipartite graphs. Canadian Journal of Mathematics, 10 517-534.

Johansson, R. \& Rantzer, A. Hybrid systems in automotive control. (2007). IEEE International Journal of Control, 80(11, Special issue).
Müller, P. C. (2000). Descriptor systems: Pros and cons of system modelling by differential-algebraic equations. Mathematics and Computers in Simulation, 53(4-6), 273-279.

Murota, K. (1987). System Analysis by Graphs and Matroids. SpringerVerlag. New York, U.S.A.

Reinschke, K. J. (1988). Multivariable Control. A Graph Theoretic Approach.. Springer-Verlag. New York, U.S.A.

\section{Appendix A: Proof of Lemma 5}

i. We can always reorder the strongly connected components to have $\mathcal{C}_{j}\left(\bar{E}_{q}\right) \preccurlyeq \mathcal{C}_{i}\left(\bar{E}_{q}\right)$ only when $i \leq j$. In this case, DulmageMendelsohn decomposition of the bipartite graph related to matrix $\tilde{E}_{q}$ leads (after a possible permutation of rows and columns of this matrix) to the following bloc decomposition of $\tilde{E}_{q}$ :

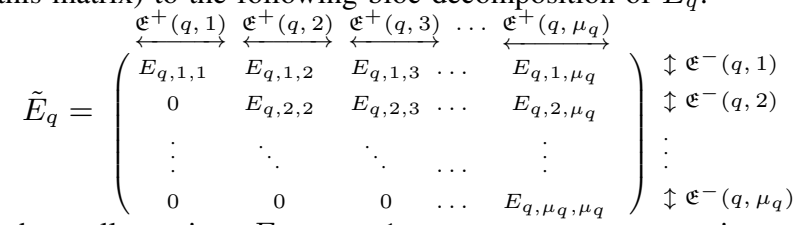

where all matrices $E_{q, i, i},=1, \ldots, \mu_{q}$ are square matrices and generically full row-rank because there is no inconsistent part in the bipartite graph related to matrix $\tilde{E}_{q}$ and $E_{q, i, j} \neq 0$ only if there exists an edge from $\mathcal{C}_{i}\left(\bar{E}_{q}\right)$ to $\mathcal{C}_{j}\left(\bar{E}_{q}\right)$. Applying the inversion triangular block matrices, to the inversion of $\tilde{E}_{q}$, we have that $\tilde{E}_{q}^{-1}$ is also a block triangular matrix. Since, $E_{q, i, i}$ is associated to a strongly connected component, this sub-matrix cannot, by any row or column permutation, be partitioned into a blocktriangular matrix. Therefore, the bipartite graph representing this matrix contains edges from all vertices of $\mathfrak{E}^{-}(q, i)$ to the ones of $\mathfrak{E}^{+}(q, i)$. Moreover, if there exists in $E_{q, i, i}$ an element which is specific to mode $q$, then all the elements of $E_{q, i, i}^{-1}$ will be specific to mode $q$ and so must be represented by edges with index $q$. Let us look now to the sub-matrices in the upper part of $\tilde{E}_{q}^{-1}$ and consider first the two last blocks of $\tilde{E}_{q}$ i.e. $\left(\begin{array}{cc}E_{q, \mu_{q}-1, \mu_{q}-1} & E_{q, \mu_{q}-1, \mu_{q}} \\ 0 & E_{q, \mu_{q}, \mu_{q}}\end{array}\right)$. The inversion of this sub-matrix gives $\left(\begin{array}{cc}E_{q, \mu_{q}-1, \mu_{q}-1}^{-1} & -E_{q, \mu_{q}-1, \mu_{q}-1}^{-1} E_{q, \mu_{q}-1, \mu_{q}} E_{q, \mu_{q}, \mu_{q}}^{-1} \\ 0 & E_{q, \mu_{q}, \mu_{q}}^{-1}\end{array}\right)$. Matrix $E_{q, \mu_{q}-1, \mu_{q}-1}^{-1} E_{q, \mu_{q}-1, \mu_{q}} E_{q, \mu_{q}, \mu_{q}}^{-1}$ is generically non-zero iff there exists an edge from $\mathcal{C}_{\mu_{q}-1}\left(\bar{E}_{q}\right)$ to $\mathcal{C}_{\mu_{q}}\left(\bar{E}_{q}\right)$. In this case, the bipartite representing this matrix contain edges from all vertices of $\mathfrak{E}^{-}\left(q, \mu_{q}\right)$ to the ones of $\mathfrak{E}^{+}\left(q, \mu_{q}-1\right)$. Moreover, if there exists a path from a vertex of $\mathcal{C}_{\mu_{q}-1}\left(\bar{E}_{q}\right)$ to a vertex of $\mathcal{C}_{\mu_{q}}\left(\bar{E}_{q}\right)$, then all the elements of $E_{q, \mu_{q}-1, \mu_{q}-1}^{-1} E_{q, \mu_{q}-1, \mu_{q}} E_{q, \mu_{q}, \mu_{q}}^{-1}$ will be specific to mode $q$ and so must be represented by edges with index $q$. We can generalize this reasoning to the other blocs of $\tilde{E}_{q}^{-1}$ and we have that the $\Gamma_{q}$-edges as defined in Subsection 4.2 corresponds generically to the edges of matrix $\tilde{E}_{q}^{-1}$, where $\tilde{E}_{q}$ is the square generically full column matrix described by the bipartite graph restricted to $\bar{E}_{q}$-edges between vertices included in $\mathfrak{E}^{+}(q, k), k \neq 0$ and the ones included in $\mathfrak{E}^{-}(q, k), k \neq 0$. Moreover, when the index of an edge of $\Gamma_{q}$-edges is equal to $q$, then the corresponding element of $\tilde{E}_{q}^{-1}$ is specific to mode $q$.

ii. According to the previous characterization of $\tilde{E}_{q}$ and as edges related to $\hat{E}_{q}$ are the ones starting from $\mathfrak{E}^{+}(q, 0)$, we have that the non zero elements of $\tilde{E}_{q}^{-1} \hat{E}_{q}$ correspond in $\bar{B}_{q, M}$ to the paths starting from $\mathfrak{E}^{+}(q, 0)$ by necessarily an $\hat{E}_{q}$-edge and arriving to some $\mathcal{C}_{i}\left(\bar{E}_{q}\right)$. This is traduced by the definition of $\Psi_{q}$-edges. If, besides, this path contains an edge with index $q$, then all the edges from the corresponding begin vertex and all vertices of $\mathcal{C}_{i}\left(\bar{E}_{q}\right)$ describe relations which are specific to mode $q$ and must have index $q$. 